\title{
Prevenção da Nefropatia induzida por contraste em pacientes submetidos a procedimentos em cardiologia intervencionista e hemodinâmica
}

\author{
Prevention of contrast-induced Nephropathy (nic) in patients submitted \\ to procedures in interventional cardiology and hemodynamics
}

LUIZA Venturini HeLAeHIL ${ }^{1}$ Gabriela Angelica Palmieri ${ }^{\mathrm{I}}$ NicásSIa SENHORINI MAgalHães ${ }^{1}$ Clarice Santana Milagres ${ }^{\mathrm{I}}$ ${ }^{\mathrm{I}}$ Centro Universitário da Fundação Hermínio Ometto (FHO). Araras/ SP - Brasil
RESUMo Estudos apontam que as doenças cardiovasculares são as principais causas de incapacidade e morbimortalidade no mundo. Nessas doenças, o transporte necessário de sangue para o músculo cardíaco não chega de maneira adequada. Suas principais complicações são angina e infarto agudo do miocárdio, sendo necessário nessa fase procedimentos para diagnosticar e até mesmo tratar a lesão. Nos últimos anos, houve grandes avanços da medicina para o tratamento dessas complicações, por intermédio dos serviços de hemodinâmica e cardiologia intervencionista, onde são realizados procedimentos como o cateterismo cardíaco para o diagnóstico e a angioplastia coronária para tratamento das obstruções das artérias coronárias. São considerados procedimentos seguros, porém, para a visualização e tratamento das artérias coronárias que ocorrem por meio de imagens radiológicas e com uso de contraste iodado endovenoso existem riscos. O contraste pode se tornar nefrotóxico para alguns pacientes que se encaixam no grupo de risco para o desenvolvimento da Nefropatia Induzida por Contrate (NIC). Cabe à equipe de enfermagem estar preparada para esse cuidado e prevenção. O presente estudo tem por objetivo verificar na literatura recente informações da cardiologia intensivista em relação aos procedimentos, métodos e diagnósticos que requerem o uso de contraste iodado, assim como, verificar a Nefropatia Induzida por Contraste em relação aos conceitos, à epidemiologia, aos diagnósticos, aos tratamentos e aos cuidados de enfermagem nesse tipo de nefropatia. Foram feitas revisões de literatura de artigos publicados entre 2012 e 2019, nas bases de dados eletrônicos PubMed Medline, Scielo e Science Direct. Foram utilizados os seguintes descritores da saúde em inglês e em português: nefropatia, "nefropatia crônica", hemodinâmica, "meios de contraste" e enfermagem usados em combinação. Além dos artigos foram revisados livros que fornecem informações a respeito do assunto publicado nos últimos dez anos. As referências bibliográficas dos trabalhos identificados pelas pesquisas eletrônicas foram revisadas para identificação de estudos adicionais. Considerações finais: é necessário identificar o grupo de risco dos pacientes que podem desenvolver NIC, a fim de aplicar as medidas de prevenção, evitando assim o surgimento dessa nefropatia. As ações de prevenção e cuidados diretos devem ser planejados para uma assistência de enfermagem qualificada, especializada e individualizada para evitar a NIC, assim como, para reduzir a incidência dessa complicação. Dessa 
forma, as informações poderão subsidiar maiores conhecimentos acerca dos grupos de riscos envolvidos nessa patologia, medidas de prevenção necessárias e planejadas, e cuidados específicos de enfermagem diante dessa situação.

Palavras-chave: Nefropatia. "Nefropatia crônica". Hemodinâmica. "Meios de contraste". Enfermagem.

Abstract Studies show that cardiovascular diseases are the main causes of disability and morbidity and mortality worldwide. In these diseases, the necessary transport of blood to the heart muscle does not arrive properly. Its main complications are angina and acute myocardial infarction, and it is necessary at this stage to diagnose and even treat the injury. In recent years, there have been great advances in medicine for the treatment of these complications, through the services of hemodynamics and interventional cardiology, where procedures such as cardiac catheterization for diagnosis and coronary angioplasty are performed to treat coronary artery obstructions. They are considered safe procedures, however, for the visualization and treatment of coronary arteries, that occur through radiological images and with the use of intravenous iodinated contrast, there are risks. The contrast can become nephrotoxic for some patients who fall into the risk group for the development of Contract-Induced Nephropathy (CIN). The nursing team must be prepared for this care and prevention. The present study aims to verify in the recent literature information on intensive care cardiology in relation to the procedures, methods and diagnoses that require the use of iodinated contrast, as well, as to verify Contrast-Induced Nephropathy in relation to concepts, epidemiology, diagnoses, nursing treatments and care in this type of nephropathy. Literature reviews of articles published between 2012 and 2019 were carried out in the electronic databases PubMed Medline, Scielo and Science Direct. The following health descriptors in English and Portuguese were used: nephropathy, "chronic nephropathy", hemodynamics, "contrast media" and nursing used in combination. In addition to the articles, books that provide information on the subject published in the last 10 years have been reviewed. The bibliographic references of the works identified by the electronic searches were revised to identify additional studies. Final considerations: it is necessary to identify the risk group of patients who may develop CIN, in order to apply preventive measures, thus preventing the appearance of this nephropathy. Prevention and direct care actions must be planned for qualified, specialized and individualized nursing care to avoid CIN, as well, as to reduce the incidence of this complication. In this way, the information may support greater knowledge about the risk groups involved in this pathology, necessary and planned prevention measures, as well as specific nursing care in the face of this situation.

Keywords: NePHropathy. "Chronic NePhropathy". HemodynamiCs. “Contrast media”. Nursing. 


\section{INTRODUÇÃO}

As doenças cardiovasculares são consideradas as principais causas de morbidade e mortalidade em todo o mundo, sendo responsável por mais de 15,9 milhões de óbitos. ${ }^{1}$ No Brasil, 300 mil pessoas morrem anualmente de doenças cardiovasculares, sendo a doença arterial coronária a principal causa de morte. ${ }^{2}$

As maiores prevalências das doenças cardiovasculares são encontradas na Doença Arterial Coronária (DAC) estável e nas síndromes coronárias agudas. Esse panorama epidemiológico demonstrou nos últimos anos crescente importância do conhecimento acerca da cardiologia intervencionista. Esta, por sua vez, tem apresentado um grande avanço, sendo o método de tratamento do miocárdio mais empregado nos cenários clínicos. ${ }^{3}$

A utilização de diversos meios diagnósticos, importantes para a definição de terapêutica adequada, é essencial. Entre eles, a tomografia computadorizada (TC) tem sido um exame radiológico de ampla indicação, no entanto, apresenta riscos associados à exposição, à radiação e ao meio de contraste iodado. $\mathrm{O}$ último procedimento deve ser realizado em ambiente laboratorial de hemodinâmica, uma vez que uma atenção especial precisa ser dada ao uso desse meio de contraste, visto que pode ocasionar Nefropatia Induzida por Contraste (NIC), já que se trata de uma substância nefrotóxica. ${ }^{4}$

A NIC pode ser reduzida ao buscar os fatores de risco dos pacientes submetidos a exames diagnósticos com utilização de compostos químicos nefrotóxicos, assim como, reconhecer as ações de prevenção NIC em pacientes submetidos a procedimentos realizados na hemodinâmica e na cardiologia intervencionista. Diante disso, o presente estudo tem por objetivo verificar na literatura recente informações da cardiologia intensivista em relação aos procedimentos, métodos e diagnósticos que requerem o uso de contraste iodado, bem como, verificar a Nefropatia Induzida por Contraste em relação aos conceitos, à epidemiologia, aos diagnósticos, aos tratamentos e aos cuidados de enfermagem nesse tipo de nefropatia.

\section{Metodologia}

Foi realizada uma revisão de literatura de artigos publicados entre 2012 e 2017, nas bases de dados eletrônicos PubMed Medline, Scielo e Science Direct, e utilizados descritores em saúde, predeterminados em inglês e em português, abrangendo artigos que empregaram nefropatia, "nefropatia crônica", hemodinâmica, "meios de contraste" e enfermagem usados em combinação. Além dos artigos foram revisados livros que forneceram informações sobre o assunto publicados nos últimos dez anos. As referências bibliográficas dos trabalhos identificados pelas pesquisas eletrônicas também foram revisadas na identificação de estudos adicionais. Foram excluídos trabalhos anteriores a 2012 e revisões de literatura.

Entre os demais critérios para a escolha dos materiais estudados foram incluídas temáticas referentes aos serviços de hemodinâmica, Nefropatia Induzida por Contraste, grupos de risco e cuidados de enfermagem nos pacientes que requerem tais procedimentos. 


\section{REVISÃo DE LITERATURA}

\section{1 - Cardiologia intervencionista}

A cardiologia Intervencionista é considerada atualmente o método mais empregado no tratamento do miocárdio em todos os cenários clínicos, incluindo a Doença Arterial Coronária estável (DAC) e as Síndromes Coronarianas Agudas (SCAs). Tal fato decorre do grande avanço da cardiologia intervencionista nos últimos anos, pelo desenvolvimento tecnológico dos dispositivos percutâneos e pelas técnicas de tratamento e indicações a esse tratamento. ${ }^{3}$

Em relação às doenças do aparelho circulatório, estas são consideradas as principais causas que levam ao óbito em todo o mundo. Dados do Estudo Carga Global de Doença mostraram que, em 2015, as doenças cardiovasculares foram responsáveis por 256 mortes a cada 100 mil habitantes. ${ }^{5}$

As doenças cardiovasculares no Brasil são responsáveis por cerca de $20 \%$ das mortes em indivíduos acima de 30 anos de idade. ${ }^{6}$ Sendo a cardiopatia isquêmica a principal causa de mortalidade, com taxas de $8,8 \%{ }^{7}$

As doenças isquêmicas do coração foram responsáveis por 85.449 mortes, as doenças cerebrovasculares por 97.860 , e a aterosclerose por 193.309 mortes. São doenças de altos custos hospitalares, sendo possível minimizá-las com a prevenção, diagnóstico e tratamento adequado. ${ }^{6}$

Os procedimentos da cardiologia intervencionistas são realizados no ambiente da hemodinâmica, mostrando-se um serviço de alta complexidade, que requer materiais específicos e utilização de técnicas guiadas com raios $\mathrm{X}$ e contraste iodado. Os principais procedimentos na cardiologia intervencionista incluem o cateterismo cardíaco, a angioplastia coronária, Persistência do Canal Arterial (PCA), Comunicação Intra- Arterial (CIA), Comunicação Intraventricular (CIV), entre outros e demais especialidades. ${ }^{8}$

\section{Cardiologia Intervencionista: Procedimentos, Métodos e Diagnósticos que requerem o uso de Contraste Iodado}

O cateterismo cardíaco é o procedimento diagnóstico detalhado que tem por objetivo obter informações sobre as estruturas cardíacas. O exame é realizado por meio da introdução de um cateter posicionado nas artérias coronárias e no ventrículo esquerdo com auxílio de injeções de contraste e tem a finalidade de detectar a existência de obstruções nas artérias coronárias e, assim, verificar o funcionamento nas câmaras e valvas cardíacas. ${ }^{9}$ A maioria dos pacientes submetidos a esse procedimento apresenta doenças coronárias e valvares, sugestivas de tratamento por via percutânea. Além do cateterismo cardíaco para o estudo das artérias coronárias e demais segmentos, outros diagnósticos são realizados pela cardiologia intervencionista e na hemodinâmica, como o Estudo Eletrofisiológico (EEF) para diagnosticar as arritmias na área da eletrofisiologia, a arteriografia cerebral na neurocirurgia e na área da cirurgia vascular a arteriografia de membros. ${ }^{10}$

Com os avanços da cardiologia intervencionista e da hemodinâmica, a medicina tem utilizado fármacos e/ou substâncias nefrotóxicas que são essenciais no diag- 
nóstico e tratamento das doenças. $\mathrm{Na}$ hemodinâmica, na qual a cardiologia intervencionista realiza os exames de imagens e utiliza o contraste iodado, essa realidade é constantemente acompanhada. ${ }^{11}$ Tais ações, reconhecidas como procedimentos desses ambientes de alta complexidade, são executadas com auxílio de raios-X e com uso de contraste iodado endovenoso. $\mathrm{O}$ último é uma substância que é injetada por meio de um cateter radiopaco, introduzido nas artérias ou veias, e auxilia na visualização. Já a radiação requer o uso desse contraste para diferenciar certas estruturas. ${ }^{12}$

Geralmente, os meios de contraste iodado são utilizados não somente na cardiologia intervencionista, mas em diversos procedimentos, na hemodinâmica, como na Tomografia Computadorizada (TC). Esta, por sua vez, emprega a radiação $\mathrm{X}$ e é imprescindível a presença de contraste. ${ }^{13}$

Quanto ao contraste, este atinge a maior concentração de tecidos e permite uma adequada visualização das estruturas com menor efeito colateral e é considerado o mais adequado. São classificados pelo tipo de imagem que geram, via de administração, características químicas ou de acordo com o método de imagem utilizado. Além disso, são classificados por suas propriedades físico-químicas, ionicidade, osmolaridade, densidade, viscosidade e solubilidade. ${ }^{14}$

O meio de contraste iodado é geralmente administrado por via oral previamente ao procedimento e/ou por via endovenosa durante o exame. Quanto à ionicidade, podem ser classificados em iônicos ou não iônicos, nos quais o último tem menor reação no organismo por possuir baixa osmolaridade e raramente causar reações, é considerado mais seguro, porém seu custo é elevado em relação ao contraste iônico. ${ }^{14}$ Quanto à osmolaridade, os contrastes podem ser isosmolares, que são os que apresentam menor osmolaridade, ao redor de $290 \mathrm{mosm} / \mathrm{kg}$, os hiposmolares, ao redor de $600 \mathrm{mosm} / \mathrm{kg} \mathrm{e}$ os hiperosmolares, acima de 1000 mosm/ kg. ${ }^{15}$ A quantidade de contraste utilizada durante o procedimento e os fatores de risco descritos na literatura determinam a gravidade dos efeitos colaterais. Contrastes de alta osmolaridade são mais nefrotóxicos e aumentam as chances de reações adversas aos pacientes do que os de baixa osmolaridade. Quanto mais próxima a osmolaridade do contraste estiver da osmolaridade do sangue, tanto melhor será sua tolerância. ${ }^{4}$

\section{2 - Nefropatia induzida por contraste}

\section{Conceitos, Epidemiologia, Diagnósticos e Tratamento}

A NIC é a terceira causa de Insuficiência Renal Aguda (IRA) em pacientes hospitalizados, chegando a atingir $12 \%$ dos casos. Está associada aos serviços de diagnóstico por imagem na hemodinâmica e na cardiologia intervencionista. ${ }^{16}$ É considerada uma importante causa de deterioração renal aguda em pacientes hospitalizados e provocada pelo uso endovenoso de contraste iodado qualificado, portanto, como uma complicação grave na hemodinâmica, podendo resultar em aumento da morbidade e mortalidade, hospitalização prolongada e aumento de custos hospitalares. ${ }^{17}$

A complicação na função renal, denominada por Nefropatia Induzida por Contras- 
te, ocorre devido ao uso de contraste iodado em intervenções diagnósticas que requerem o uso de tal substância. É descrita como piora aguda da função renal após administração de contraste por via endovenosa, depois de se afastar outras causas, ${ }^{4}$ estando associada a altas taxas de morbimortalidade, tempo, e custos hospitalares. ${ }^{18}$

A incidência da NIC varia de acordo com os fatores associados, podendo contribuir para o aumento de $10 \%$ a $30 \%$ em sua incidência. A idade avançada, a presença de diabetes, a função renal comprometida, como Doença Renal Crônica (DRC), a creatinina prévia alterada, o tipo e a administração de altas doses de contraste, ${ }^{21}$ podem ser considerados fatores associados, assim como, a Insuficiência Cardíaca Congestiva (ICC), a hipovolemia, utilização de agentes nefrotóxicos e anti-inflamatórios não hormonais, e varia de $10 \%$ a $30 \%$ na população estudada. ${ }^{19}$

A definição mais específica da NIC é a elevação absoluta da creatinina sérica igual ou superior a $0,5 \mathrm{mg} / \mathrm{dl}$ ou aumento relativo de $25 \%$ ou mais no período de 48 a 72 horas após a administração do contraste à base de iodo e sem outras causas identificáveis. ${ }^{19}$ Está associada aos mecanismos inflamatórios, à disfunção endotelial e ao estresse oxidativo. ${ }^{17}$ Uma das teorias para as explicações da NIC seria o efeito dos radicais livres de oxigênio e estresse hiperosmolar na medula renal. ${ }^{20}$ Diversos estudos abordam a detecção e a prevenção da NIC, a fisiopatologia e o tratamento dessa patologia são incertos. Seu prognóstico é negativo e eleva a morbidade, o risco de complicações, como a necessidade de diálise, a elevação das taxas de mortalidade e o prolongamento da hospitalização, gerando altos custos. ${ }^{21}$
A partir do momento que se instala a NIC, toda uma terapêutica deve ser estabelecida, pois a instauração da IRA é a consequência mais prevalente. ${ }^{16} \mathrm{~A}$ identificação de fatores que possam prevenir o comprometimento da função renal após procedimentos que utilizam contraste iodado e o uso de protocolos direcionados à detecção precoce dos pacientes com maior risco é importante e deve ser inicializada no momento da internação que antecede esses procedimentos. ${ }^{22}$

Para a prevenção da NIC, a literatura tem apresentado dados sobre a hidratação endovenosa, uso de diuréticos, vasodilatadores, inibidores farmacológicos dos vasoconstritores e antioxidantes. ${ }^{4}$ Alguns autores argumentam que a hidratação com bicarbonato de sódio diminui a lesão renal por sua alcalinização nos túbulos renais. ${ }^{20}$ Nas diretrizes europeias, $75 \%$ dos estudos sobre NIC tiveram algum tipo de hidratação como prevenção. Assim, recomendam expansão de soro fisiológico $0,9 \%$ ou bicarbonato de sódio antes do uso do contraste. ${ }^{23}$ Em estudo comparativo entre esses produtos na hidratação via endovenosa constatou-se que o cloreto de sódio a $0,9 \%$ mostrou-se mais eficaz, enquanto a administração de bicarbonato de sódio e o baixo volume de contraste foram considerados adequados para reduzir o risco da NIC. Nesse mesmo estudo a $\mathrm{N}$-acetilcisteína, que inibe a ação de radicais livres de oxigênio, protegendo desse modo a função renal, sendo ela administrada por via oral, 24 horas antes da injeção de contraste, ${ }^{22}$ foi analisada para prevenir a NIC, no entanto não mostrou resultados satisfatórios. ${ }^{4}$ 


\section{Cuidados de Enfermagem na NIC}

A alteração da função renal deve ser precocemente diagnosticada por ser reversível, porém se não tratada pode levar o paciente ao desenvolvimento de uma deterioração renal aguda, com graves consequências. O uso de contraste é uma forma grave de iatrogenia e deve ser elucidado para a correta realização de procedimentos diagnósticos que utilizam contrastes iodados. ${ }^{4}$

O Conselho Regional de Enfermagem do Estado de São Paulo, sob o Parecer n ${ }^{\circ}$. 30, de 2014, define que todo serviço que realiza exames de imagem e diagnóstico deverá possuir uma equipe de enfermagem capacitada para a realização de uma prática segura, com conhecimento de biossegurança, a fim de prevenir, diminuir ou eliminar o risco, e quaisquer riscos inerentes às substâncias que são utilizadas em tais serviços. ${ }^{13}$ As identificações de possíveis complicações permitem reduzir desconfortos graves ao paciente, aumentar a qualidade do atendimento, reduzir gastos hospitalares e contribuir para uma assistência eficaz e integral..$^{24,25}$

A formulação de protocolos direcionados para a detecção precoce da NIC é considerada importante para o cuidado. ${ }^{22}$ É nesse cuidado que a equipe de enfermagem que atua em serviços de hemodinâmica tem um papel fundamental na assistência dos pacientes, assegurando a correta administração do contraste, de maneira que possam reconhecer os pacientes de risco para o desenvolvimento da NIC. ${ }^{2}$ A identificação de fatores de risco que possam comprometer a função renal após exames que utilizam o contraste é importante e o ideal é estabelecer critérios de cuidados e segurança do paciente antes que a deterioração renal seja instalada. Pacientes com risco para o desenvolvimento da NIC podem ser identificados na admissão, passando por consulta de enfermagem e médica, estabelecendo condutas de cuidados para a prevenção desse risco. ${ }^{24,25}$ Logo, a interação com a equipe assistencial de enfermagem, a equipe médica e o paciente são fundamentais no auxílio de um atendimento livre de complicações, no qual a equipe assistencial no laboratório de hemodinâmica deve oferecer um atendimento livre de danos, gerenciando condutas de cuidados para os pacientes com risco de desenvolvimento da NIC.

As ações de prevenção e cuidados diretos devem ser planejadas para uma assistência de enfermagem qualificada, especializada e individualizada para evitar a NIC, assim como, para reduzir a incidência dessa complicação. Ao identificar pacientes de grupos de risco para o desenvolvimento da NIC, deve-se iniciar uma estratégia de proteção renal o quanto antes, através de meios de prevenção como pré-medicação com a $\mathrm{N}$-acetilcisteína, hidratação com soro fisiológico $0,9 \%$ (solução isotônica) e solução de bicarbonato (alcalinização isotônica). Outra ação importante é que a equipe de enfermagem precisa estar atenta aos medicamentos considerados nefrotóxicos em pacientes de risco, que devem ser suspensos antes do uso do contraste, em especial em pacientes diabéticos que fazem uso da metformina; e fazer o acompanhamento de resultados dos exames laboratoriais, principalmente, 48 a 72 horas após o uso de contraste. ${ }^{10}$

Em relação aos exames de imagem e diagnóstico que utilizam contrastes ioda- 
dos em portadores de Doença Renal Crônica (DRC), é necessário assegurar uma boa hidratação antes, durante e após o uso do contraste. Também se faz necessário utilizar contrastes específicos, ou seja, contrastes não iônicos; e assegurar baixas doses durante o exame. A indicação mais adequada, e o melhor tratamento, é, portanto, a prevenção, que é realizada por meio da hidratação endovenosa pré e pós-procedimento e o uso de contrastes de baixa ou iso-osmolaridade e em baixas doses, quando necessários. ${ }^{24,25}$ Apesar da condição do cuidado especial implantado junto ao portador de DRC, cuidados semelhantes devem ser realizados para todos os pacientes que estão inseridos no grupo de risco para NIC. ${ }^{3}$

\section{CONSIDERAÇões FINAIS}

Este trabalho demonstrou ser necessário identificar o grupo de risco dos pacientes que podem desenvolver NIC, a fim de aplicar as medidas de prevenção, evitando assim o surgimento dessa nefropatia. As ações de prevenção e cuidados diretos devem ser planejadas para uma assistência de enfermagem qualificada, especializada e individualizada para evitar a NIC, assim como, para reduzir a incidência dessa complicação. Dessa forma, as informações poderão subsidiar maiores conhecimentos acerca dos grupos de riscos envolvidos nessa patologia, medidas de prevenção necessárias e planejadas, e cuidados específicos de enfermagem diante dessa situação.

\section{REFERÊNCIAS}

1. Faludi AA, Izar MCO, Saraiva JFK, Bianco HT, Chacra APM, Bertoluci MC et al. Diretriz brasileira baseada em evidências sobre prevenção de doenças cardiovasculares em pacientes com diabetes: posicionamento da Sociedade Brasileira de Diabetes (SBD), da Sociedade Brasileira de Cardiologia (SBC) e da Sociedade Brasileira de Endocrinologia e Metabologia (SBEM). Arq. Bras. Cardiol, 2017.

2. Acauam LV, Rodrigues MCS. Critérios de Segurança na administração de contraste na angiotomografia cardíaca: percepção da enfermagem. Revista Rene, Brasília, (16) 4: 504-513, 2015.

3. Feres F., Ricardo AC, Dimytri S., Ribamar CJ, Daniel C., Rodolfo S. et al. Diretriz da Sociedade Brasileira de Cardiologia e da Sociedade Brasileira de Hemodinâmica e Cardiologia Intervencionista Sobre Intervenção Coronária Percutânea. Arquivos Brasileiros de Cardiologia, (109) 1:1-81, 2017.

4. Aoki BB, Fram D., Taminato M., Batista RES, Belasco A., Barbosa DA. Acute kidney injury after contrast-enhanced examination among elderly. Revista Latino-americana de Enfermagem, [s.l.], (22) 4: 637-644, 2014.

5. Brant LCC, Nascimento BR, Passos VMA, Duncan BB, Bensenõr IJM, Malta DC et al. Variações e diferenciais da mortalidade por doença cardiovascular no Brasil e em seus estados, em 1990 e 2015: estimativas do Estudo Carga Global de Doença. Rev. bras. Epidemiol, (20) 1: 116-128, 2017.

6. Mansur AP, Favarato D. Trends in Mortality Rate from Cardiovascular Disease in Brazil, 19802012. Arquivos Brasileiros de Cardiologia, [s.l.], 20-27, 2016.

7. Araujo GN, Valle FH, Machado GP, Pivatto F., Fuhr B., Cassol EP et al. Desfechos cardiovasculares em pacientes tratados com intervenção percutânea coronária primária em hospital geral terciário. Revista Brasileira de Cardiologia Invasiva, [s.l.], (24) 4: 4-8, 2016. 
8. Flôr RC, Gelbcke FL. Proteção radiológica e a atitude de trabalhadores de enfermagem em serviço de hemodinâmica. Texto \& Contexto - Enfermagem, [s.l.], (22) 2: 416-422, 2013.

9. Secco AC, Scortegana SA, Tognon AP, Espíndola AV, Benincá C. et al. Intervenção psicológica vídeo-orientativa em pacientes submetidos ao cateterismo cardíaco. Revista da Pucrs, Porto Alegre, (48) 3: 205-2015, 2017.

10. Padilha, Katia Grillo et al. (Org.). Enfermagem em UTI: cuidados do paciente crítico. São Paulo: Manole Ltda, 1446 p., 2010.

11. Vattimo MFF, Santos JG. O efeito protetor do bicarbonato de sódio na NICradiológico em ratos. Revista da Escola de Enfermagem da Usp, [s.1.], (47) 3: 722-727, 2013

12. Moreira MLAP, Mizuno E., Meireles GCX. Consulta de enfermagem pré-cateterismo cardíaco e intervenções coronárias percutâneas. Revista de Enfermagem Ufpe On Line, Recife, 2.5482.556, 2017.

13. CONSELHO REGIONAL DE ENFERMAGEM DE SÃO PAULO (Estado). Parecer COREN-SP $\mathbf{n}^{\mathbf{0}}$. 030/2014. Administração de meios de contraste em setor de imagem e diagnóstico.

14. Sartori P., Rizzo F., Taborda N., Anaya V., Caraballo A., Saleme C. et al. Mídia de contraste em imagens. Rev. argent. radiol. Cidade Autônoma de Buenos Aires, (77) 1: 49-62, 2013.

15. Romano TG, Tierno PFGM. Acute Renal Injury in polytrauma patients. Jornal Brasileiro de Nefrologia, [s.l.], (35) 1: 48-56, 2013.

16. Morales JLF. Actualidad en nefropatía por medio de contraste. Nefrología Latinoamericana, [s.l.], (14) 2: 69-78, 2017.

17. Calça R., Teles RC, Branco P., Gaspar A., Brito J., Nolasco T. et al. Impacto do Implante Percutâneo de Válvula Aórtica na Função Renal. Arq. Bras. Cardiol. Dec, 113 (6): 1.104-1.111, 2019.

18. Gomes VO. Hidratação com bicarbonato de sódio não previne a nefropatia de contraste: ensaio clínico multicêntrico. Arquivos Brasileiros de Cardiologia, [s.l.], (99) 6: 1.129-1.134, 2012.

19. Maccariello E. Contrast induced nephropathy. Jornal Brasileiro de Nefrologia, [s.1.], (38) 4 : 388-389, 2016.

20. Nieto-ríos JF et al. Prevention of contrast induced nephropathy with sodium bicarbonate (the PROMEC study). Jornal Brasileiro de Nefrologia, [s.l.], (36) 3: 360-366, 2014.

21. Santos PR, Neto JDC, Arcanjo FPN, Carneiro JKR, Carneiro RCCP, Amaral CL. NICapós angioplastia primária no infarto agudo do miocárdio. Jornal Brasileiro de Nefrologia, Sobral, (37) 4: 439-445, 2015.

22. Moura ELB, Amorim FF, Huang W, Maia MO. Contrast-induced acute kidney injury: the importance of diagnostic criteria for establishing prevalence and prognosis in the intensive care unit. Revista Brasileira de Terapia Intensiva, [s.l.], (29) 3: 303-309, 2017.

23. Selistre LS, Souza VC, Dubourg L., Wagner MB, Filho JRH, Saitovitch D. Contrast-induced nephropathy after computed tomography. Jornal Brasileiro de Nefrologia, [s.l.], (37) 1: 27-31, 2015.

24. Santos AFS, Silva IB, Carvalho SQS, Junior CVA. Assistência de Enfermagem a Pacientes Submetidos à Angioplastia Coronária: Uma Revisão de Literatura. Ciências Biológicas e da Saúde UNIT: Ciências biológicas e da saúde, Alagoas, (4) 1: 191-201, 2017.

25. Centemero MP, Sousa AGMR. Predição de nefropatia induzida pelo contraste após revascularização coronária percutânea: precisamos de formular? Uma perspectiva da cardiologia. Revista Portuguesa, São Paulo, 25-33, 2018. 


\section{DADOS DAS AUTORAS}

\section{Gabriela Angelica Palmieri}

Enfermeira especialista em Nefrologia pelo Centro Universitário da Fundação Hermínio Ometto. Araras/SP - Brasil.gabrielaangelica_palmieri@hotmail.com

\section{Nicássia Senhorini Magalhães}

Enfermeira especialista em Terapia Intensiva: Cardiologia pelo Centro Universitário da Fundação Hermínio Ometto. Araras/SP - Brasil.nicassiasmagalhaes@gmail.com

\section{Luiza Venturini Helaehil}

Graduanda em enfermagem no Centro Universitário da Fundação Hermínio Ometto. Araras/SP Brasil. luizahelaehil@gmail.com

\section{Clarice Santana Milagres}

Doutora em odontologia/Saúde Coletiva pela Universidade Estadual de Capinas/Faculdade de Odontologia de Piracicaba (FOP/UNICAMP). Docente de enfermagem no Centro Universitário da Fundação Hermínio Ometto. Araras/SP - Brasil. claricemilagres01@gmail.com

Submetido em: 1-7-2018

Aceito em: 22-6-2020 